\title{
Protéinogramme des sérums de zébus Gobra au Sénégal. Variations quantitatives saisonnières
}

\author{
par R. BOUDERGUES (*) et H. CALVET $\left(^{*}\right)$
}

\begin{abstract}
RESUME
Dans un premier temps expérimental, les techniques d'électrophorèse sur acétate de cellulose utilisées en médecine humaine ont été adaptées à l'étude de sérums bovins. La détermination de nouvelles constantes opératoires a alors permis d'obtenir la séparation nette des principales fractions qui donnent à l'Intergraph $\mathrm{EL} /$ phor 4 pics nettement distincts et quantifiables.

Deux séries de prélèvements ont alors été effectuées sur des troupeaux de zébus vivant dans la zone sahélienne du Sénégal. La première a lieu en janvier, époque qui marque dans le milieu naturel la fin de la période favorable pour l'alimentation des troupeaux. La deuxième en juillet, en fin de saison sèche au moment où les animaux sont soumis à une disette générale.

La comparaison statistique de ces deux séries de sérum montre que de janvier à juillet, le taux des protéines totales subit une sensible diminution à laquelle participent en valeur absolue toutes les fractions. Leur valeur relative, par contre, subit des évolutions différentes. C'est ains1 qu'en saison sèche, le pourcentage des albumines et des $\gamma$ globulines a tendance à augmenter tandis que diminue très sensiblement celui de $\alpha$ et $\beta$ globulines.
\end{abstract}

L'étude des protéines sériques chez plusieurs espèces animales a fait l'objet, ces dernières années, d'un certain nombre de travaux réalisés dans différentes régions tropicales.

C'est ainsi que LABOUCHE, dès 1959, souligne les difficultés rencontrées au cours des électrophorèses pour obtenir une bonne séparation des diverses fractions avec les sérums bovins et tout spécialement avec ceux des bovins tropicaux. Des erreurs dans l'interprétation quantitative des courbes densitométriques peuvent en résulter et cet auteur propose alors une méthode mathématique pour l'interprétation des électrophorégrammes.

(*) I.E.M.V.T., Laboratoire national de l'Elevage et de Recherches vétérinaires, B.P. n² 2057 DakarHann.
En 1962, GIDEL (1), effectuant des électrophorèses en gélose sur des sérums de zébus et de taurillons de Haute-Volta, montre qu'il existe des différences notables dans les protéinogrammes de ces deux espèces. Les plus importantes portent sur les $\beta$ globulines dont le pourcentage est nettement augmenté chez les taurins.

PROVOST, BORREDON et QUEVAL (5), en 1965, au cours d'enquêtes sérologiques sur des troupeaux du Tchad, rencontrent un certain nombre de sérums provenant de bovins adultes, vaccinés plusieurs fois contre la peste, sur lesquels ils ne décèlent, cependant, aucun anticorps. Les électrophorèses et immunoélectrophorèses de ces sérums anormaux ne révèlent, dans le premier cas, aucun pic attribuable aux $\gamma$ globulines et, dans le second, 
aucun arc appartenant à cette fraction. Pour ces auteurs, il existe donc chez certains animaux une hypogamma-globulinémie essentielle.

Plus tard, en 1967, QUEVAL, GRABER et Mme BRUNET, étudiant les constantes hématologiques des camélidés du nord Tchad, soulignent les variations de la protidémie en fonction de l'intensité du parasitisme. Après un traitement anthelminthique énergique, apparaît une rapide augmentation des protéines totales et des modifications du protéinogramme se traduisant par: un accroissement important des albumines, une augmentation plus légère des $\beta$ globulines, tandis que diminuent les $u$ et $\gamma$ globulines.

Ces quelques travaux réalisés en zone tropicale soulignent les divers intérêts de ce type d'études. En effet, les protéinogrammes sériques sont susceptibles d'apporter d'utiles enseignements en sérologie, en pathologie et même en zootechnie, en raison d'une certaine spécificité des images électrophorétiques dans le cadre de la race et de l'espèce.

Cependant, un problème semblait n'avoir pas été envisagé à ce jour : celui des rapports existant entre la répartition des différentes fractions protéiques et l'état nutritionnel des animaux. C'est ce dernier aspect qui a été envisagé dans une série de travaux entrepris récemment au Laboratoire de Recherches vétérinaires de Dakar.

Dans un premier temps, ROUMEGOUX et DOUTRE (7), à la suite d'une adaptation des techniques, parviennent à présenter une image immuno-électrophorétique du sérum de zébu Gobra, comportant 15 arcs de précipitations dont la plupart sont identifiés avec certitude, tout particulièrement ceux correspondant aux immuno-globulines (IgA, IgG et IgM.)

Ces auteurs entreprennent ensuite une étude qualitative comparée sur deux séries de sérums prélevés sur des zébus du nord Sénégal. La première, à l'issue d'une mission effectuée en fin de saison des pluies, moment où l'état nutritionnel des animaux est le meilleur; la deuxième en fin de saison sèche, qui correspond à une période de grave disette pour les troupeaux. Les résultats ne font apparaître aucune différence d'ordre qualitatif sur ces deux groupes de sérums. On retrouve, en particulier, sur tous les animaux, les trois immuno-globulines (IgA, IgG, IgM).
Le deuxième temps de ces recherches correspond au travail qui est ici présenté. Son objet est l'étude des variations saisonnières des différentes fractions protéiques effectuées à partir des deux mêmes séries de sérums.

Les points suivants seront successivement envisagés :

- Mise au point de la technique utilisée pour ces électrophorèses;

- Présentation des résultats pour les deux groupes d'animaux;

- Analyse de ces résultats et conclusion.

\section{MISE AU POINT D'UNE TECHNIQUE D'ELECTROPHORESE}

La séparation des différentes fractions protéiques du sérum de bovin par électrophorèse sur papier, s'est toujours heurtée à des difficultés techniques.

LABOUCHE (3) n'a pu isoler les $\beta$ et $\gamma$ globulines. IRFAN (2) obtient par contre, entre les $\beta$ et les $\gamma$ globulines, un pic très proche des $\beta$ qu'il nomme une $\gamma_{1}$ globuline; cet auteur signale aussi que les a globulines migrent en une seule fraction qu'il considère comme la somme de $\alpha_{1}$ et $\alpha_{2}$ globulines.

L'électrophorèse sur acétate de cellulose comme support, introduite par KOHN en 1957, constitue un net progrès, car ce nouveau matériau, tout en procurant des conditions opératoires bien plus rapides, donne une séparation très fine des différentes fractions sériques. L'absorption du colorant par l'acétate de cellulose étant pratiquement nulle, on obtient sur un fond transparent des bandes colorées facilement intégrables par un appareil à absorption.

En médecine humaine, les techniques de séparation des différentes fractions protéiques sont maintenant bien codifiées, mais ces méthodes ne sont pas directement transposables à l'étude des sérums bovins. Les protéines animales, en effet, diffèrent des protéines humaines par leur poids moléculaire, leur $\mathrm{pH}$ isoélectrique, leur vitesse de migration et il a donc été nécessaire de déterminer de nouvelles constantes opératoires. 


\section{A. Matériel et méthodes}

\section{Sérum}

Le sang est prélevé aseptiquement à la jugulaire. Après coagulation, le sérum est centrifugé et réparti, additionné de quelques microcristaux de Merseptyl, en ampoules scellées. Après ce conditionnement, les sérums sont stockés à $-20^{\circ}$ et décongelés au moment de leur utilisation.

\section{B. Electrophorèse}

Sur un pool de sérum de bovin, des essais systématiques de différents tampons ont été effectués en faisant varier pour chacun le $\mathrm{pH}$, le voltage et la durée de migration.

Les meilleures séparations de fractions ont été obtenues pour les conditions opératoires suivantes :

\section{Tampon}

- Tris (hydroxyméthyl) aminométhane . . . . . .

— Ethylène diamine tétracétique disodique . . . . . . . 7,82 g

- Acide borique . . . . . $4,6 \mathrm{~g}$

- Acétate de calcium . . . $1,0 \mathrm{~g}$

— Eau distillée q.s.p. . . . . $1.000 \mathrm{ml}$

Le $\mathrm{pH}$ de cette solution est ajusté à 9,2 par addition de quelques gouttes de soude.

\section{Support}

- Acétate de cellulose "Cellogel " (1);

- Bande de $2,5 \times 17 \mathrm{~cm}$ ou $4 \times 17 \mathrm{~cm}$;

- Cuve Shandon;

- Portoir de $11 \mathrm{~cm}$.

Le dépôt est effectué à $1,5 \mathrm{~cm}$ du côté cathodique avec le semi-micro applicateur Chemetron.

1,5 microlitre de sérum est déposé sur $9 \mathrm{~mm}$ de long.

Un courant de 110 volts est appliqué pendant 3 heures et entraîne une migration de $6 \mathrm{~cm}$ environ de la fraction la plus mobile.

Les bandes sont ensuite colorées à l'Amidoschwartz, "transparisées" dans un bain au

(1) Cellogel Chemetron, Société SEBIA, 2, Bd Exelmans, Paris (16*).
Diacétone-Alcool, séchées et passées à la lecture pour évaluation quantitative des différentes fractions dans un Integraph Elphor $\left({ }^{2}\right)$ utilisant le filtre orange à $487 \mathrm{~m} \mu$.

\section{Résultats}

On constate sur la bande de Cellogel " transparisée ", la présence de 4 fractions parfaitement individualisées qui correspondent, par ordre de migration décroissante, aux albumines puis aux $\alpha, \beta$ et $\gamma$ globulines. La détermination qualitative de chaque fraction résulte de la comparaison sur une même bande de Cellogel $(4 \times 17 \mathrm{~cm}$ ), d'un pool de sérum bovin et d'un échantillon témoin de fraction globuline pure d'origine bovine $\left({ }^{3}\right)$.

$\alpha$ globuline fraction IV B grade

$\beta$ globuline fraction III B grade

$\gamma$ globuline fraction II B grade

Le tracé d'enregistrement de l'Integraph Elphor montre 4 pics nettement séparés et, dans la fraction $\alpha$ globuline, un point d'inflexion qui permet de différencier les $\alpha_{1}$ des $\alpha_{2}$ globulines (schéma 1). Avec certains sérums, on note en plus un deuxième point d'inflexion dans les pics des $\gamma$ globulines (schéma 2). Par comparaison avec la fraction II $\gamma$ globuline bovine pure et la fraction III $\beta$ globuline pure, ce deuxième pic se situe toujours dans la partie des $\gamma$ globulines. Lors du dosage quantitatif de ces tracés électrophorétiques, cette fraction a été intégrée avec les $\gamma$ globulines.

\section{VARIATIONS SAISONNIERES}

Deux séries de prélèvements ont été effectuées dans le Ferlo sur des troupeaux de zébus. La première en fin de saison des pluies (novembre 1969) et la deuxième en fin de saison sèche (mai 1970).

Dans chaque série, 54 prélèvements furent pris au hasard pour dosage des protéines totales et électrophorèse des sérums.

(2) Integraph Elphor, type ES 60 du Dr Bender et Dr Hobein Munich, Labomoderne, Paris (15).

(i) Calbiochem Laboratoires Eurobio, 20, Bd SaintGermain, Paris $\left(5^{\circ}\right)$. 


\section{RESULTATS}
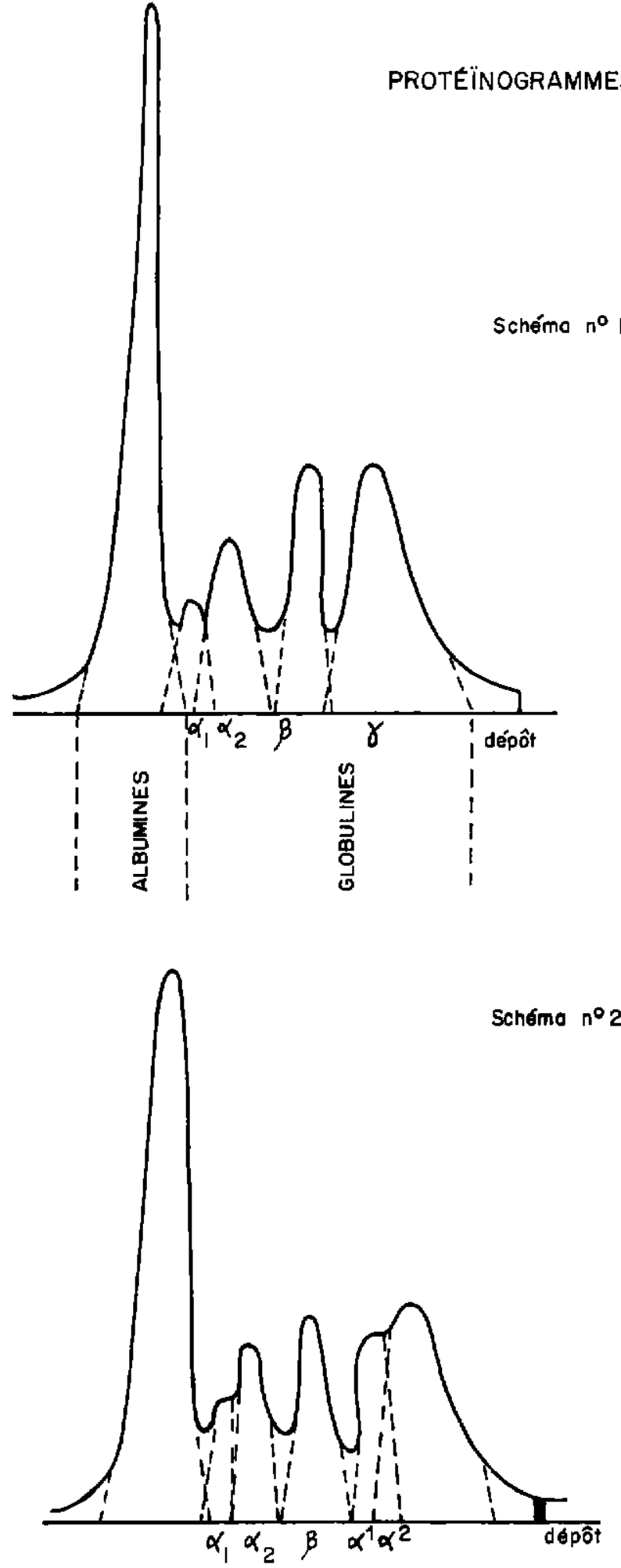

\section{Protéines totales}

En fin de saison humide, le taux des protéines totales est de $88,2 \mathrm{~g} /$ litre $\pm 2,7$ (intervalle de confiance à 5 p. 100) alors qu'en fin de saison sèche, il est de $72,7 \mathrm{~g} /$ litre $\pm 4,2$. Cette différence est hautement significative. $F=38,2$; il faut aussi noter la plus grande variabilité de ce résultat en fin de saison sèche $\pm 4,2$ qu'en saison humide 2,7 (Tableau $\mathbf{n}^{\circ} \mathrm{I}$ ).

\section{Fractions sériques}

La comparaison des résultats obtenus en janvier et en juillet est effectuée, d'abord à partir des données correspondant aux valeurs absolues de chacune des fractions (exprimées en $g$ par litre), ensuite à partir de leur valeur relative correspondant au pourcentage de la fraction par rapport à l'ensemble.

\section{Valeur absolue des fractions}

$\mathrm{Au}$ cours de la saison sèche, il apparaît une diminution des protéines totales, et un abaissement des taux de chacune des fractions.

Les différences entre les données obtenues en janvier et en juillet sont, dans tous les cas, hautement significatives, mais les valeurs de $\mathrm{F}$ indiquent qu'elles sont plus sensibles pour les $\alpha_{1}, \alpha_{2}$ et $\beta$ globulines $(F=68,1-96,9-62,2$ ) que pour les albumines et les $\gamma$ globulines $(\mathrm{F}=7,79$ et 11,1$)$.

Valeur relative des fractions

Au cours de la diminution générale des taux qui se produit en fin de saison sèche, les fractions sériques se comportent différemment en pourcentage de l'ensemble.

C'est ainsi qu'on note, en saison sèche et par rapport à janvier, une augmentation relative des albumines et de $\gamma$ globulines. Les albumines qui, en janvier, représentent 36,7 p. 100 des protéines totales, passent, en juillet, à 39,7 p. 100 et subissent donc une augmentation relative de 8 p. 100 . Les globulines se comportent de la même façon en augmentant de 4,8 p. $100(37,8$ p. 100 à 39,6 p. 100$)$.

La progression en saison sèche de ces deux 
TABLEAU $\mathrm{N}^{\circ}$ I

Valeur quantitative des fractions protéiques en g/litre et en p.100

\begin{tabular}{|c|c|c|c|c|c|c|}
\hline \multirow{2}{*}{\multicolumn{2}{|c|}{ Pratéines totales }} & \multirow[b]{2}{*}{$\mathrm{g} / 1$} & \multicolumn{2}{|c|}{ Janvier } & \multicolumn{2}{|c|}{ Juillet } \\
\hline & & & $88,2 \quad \mathrm{~g}$ & $g / 1 \pm 2,7$ & 72,7 & $\mathrm{~g} / 1 \pm 4,2$ \\
\hline \multirow{2}{*}{\multicolumn{2}{|c|}{ Albumines }} & $\mathrm{g} / 1$ & 32,3 & $" \pm 1,6$ & 28,9 & $" \pm 1,8$ \\
\hline & & $\mathrm{p} \cdot 100$ & 36,7 & $" \pm 1,74$ & 39,7 & $" \pm 1,09$ \\
\hline \multirow{10}{*}{ Globulines } & & $g / 1$ & 4,23 & $" \pm 0,36$ & 2,52 & $" \pm 0,21$ \\
\hline & & p. 100 & 4,8 & $" \pm 0,38$ & 3,6 & $" \pm 0,28$ \\
\hline & & $\mathrm{g} / 1$ & 8,61 & $" \pm 0,51$ & 5,5 & $" \pm 0,42$ \\
\hline & & p. 100 & 9,8 & $" \pm 0,50$ & 7,4 & $" \pm 0,30$ \\
\hline & & $\mathrm{g} / 1$ & 12,8 & $" \pm 0,64$ & 7,8 & $" \pm 0,54$ \\
\hline & & p. 100 & 14,1 & $" \pm 0,96$ & 10,8 & $" \pm 0,54$ \\
\hline & & $\mathrm{g} / 1$ & 9,6 & $" \pm 0,56$ & 6,9 & $" \pm 0,45$ \\
\hline & & p. 100 & 10,88 & $" \pm 0,56$ & 9.5 & $" \pm 0,52$ \\
\hline & & $g / 1$ & 33,4 & $" \pm 2,0$ & 28,8 & $" \pm 2,0$ \\
\hline & & $\mathrm{p} .100$ & 37,8 & $" \pm 1,77$ & 39,6 & $"+1,1$ \\
\hline
\end{tabular}

fractions est compensée, par contre, par un abaissement relatif important des $\alpha$ et $\beta$ globulines qui diminuent alors les unes de 23,4 p. 100 , les autres de 12,8 p. 100 .

\section{CONCLUSIONS}

Il est donc possible, par une bonne adaptation des techniques d'électrophorèse utilisées en clinique humaine, d'obtenir une nette séparation des différentes fractions protéiques des sérums bovins. Ces dernières peuvent être aisément quantifiées.

Des études ont été conduites, en saison d'élevage encore favorable (janvier) et en fin de saison sèche (juillet), sur les sérums provenant de deux troupeaux comparables de zébus vivant dans le nord Sénégal.

Elles permettent de constater, en saison sèche, une diminution en valeur absolue de toutes les fractions avec une répartition différente de leur pourcentage. C'est ainsi que, proportionnellement et à cette dernière époque, les albumines et les $\gamma$ globulines augmentent tandis que les $\alpha$ et $\beta$ globulines subissent une diminution très sensible.

Les $\alpha$ et $\beta$ globulines semblent donc correspondre à cette " réserve protéique labile (Whipple) " que l'animal utilise en saison sèche, correspondant à une période de disette protéique.

Une homéostasie étroite tend, par contre, à maintenir autant que possible le taux des albumines, indispensables à la régulation hydrique, et celui des gamma globulines, liées au processus de défense de l'organisme.

Dans ces deux séries de recherches, ne sont donc jamais apparues les anomalies importantes, concernant une hypo ou une agammaglobulinémie, observées au Tchad par PROVOST et ses collaborateurs (5).

Un nouveau travail est en cours sur des animaux du Centre de Recherches Zootechniques de Dara qui a pour objet de suivre mensuellement l'évolution des différentes fractions sériques au cours du cycle annuel. 


\title{
SUMMARY
}

\author{
Studies on electrophoresis on cellulose acetate membrane \\ of bovine serum protein in healthy zebu cattle, during \\ two different seasons of the year
}

It has been first necessary to settle new operative constants in achieving a clearly defined separation of the various bovine serum fractions. Under these conditions, curves show four main peaks which present albumin, $\alpha$ globulin, $\beta$ globulin, $\gamma$ globulin fractions.

Two series of blood samples performed, the first in jatluary, the second in july, are obtained from zebu flocks living in the North Senegal. The comparison of the results shows that the total serum proteins and each of their fractions are lower $(\mathrm{g} / 1)$ at the end of the dry season. However the per cent of the four main fractions changes from one to the other season. In the dry one, albumin and globulin are proportionally increased while $\alpha$ and $\beta$ globulins are sharply reduced.

\section{RESUMEN}

\section{Proteinograma de los sueros de cebues Gobra en Senegal. Variaciones cuantitativas de temporada}

Durante una primera fase experimental, se adaptaron las técnicas de electroforesis sobre acetata de celulosa utilizadas en medicina humana para el estudio de sueros de bovinos. La determinación de nuevas constantes operatorias permitió obtener la separación neta de las principales fracciones que dan, mediante el Integraph Elphor, cuatro vertices muy distintos.

Se tomaron dos series de muestras sobre cebues viviendo en la zona saheliana de Senegal: la primera en enero, mes que indica, en el ambiente natural el fin del periodo favorable para la alimentación del ganado, la segunda en julio, al fin de la estación seca cuando los animales padecen con una carestía general.

La comparación estadística de dichas dos series muestra que de enero a julio, la tasa de proteinas totales disminuye notablemente asi como todas las fracciones en valor absoluto. En cambio su valor relativo evoluciona diferentemente. Durante la estación seca es cuando tiende a aumentar el porcentage de las albuminas y de las $\gamma$ globulinas mientras que disminuye muy notablemente el de $\alpha$ y $\beta$ globulinas.

\section{BIBLIOGRAPHIE}

1. GIDEL (R.), Etude électrophorétique quantitative en gélose des protéines sériques de bovins, $R e v$. Elev. Méd. vét. Pays trop., 1962, 15 (3) : 259-63.

2. IRFAN (M.), The electrophoretic pattern of serum proteins in normal animals, Res. vet. Sci., 1967, 8 (2): $137-42$.

3. LABOUCHE (C.), Bases mathématiques d'une interprétation éventuelle des séparations électrophorétiques incomplètes, Ann., Biol, anim. Bioch. Biophys., 1962, 2 (4) : 319-28.

4. NILS (E. K.), Study on electrophoresis on cellulose acetate membrane of bovine serum proteins in healthy animals, Acta vet. scand., 1969, 10: 118-26.
5. PROVOST (A.), BORREDON (C.), QUEVAL (R.), Une hypogammaglobulinémie essentielle des bovins d'Afrique centrale, cause d'erreur dans les enquêtes sérologiques, Rev. Elev. Méd. vét. Pays trop., 1965,18 (4) : 385-93.

6. QUEVAL (R.), Contribution à l'étude quantitative des protéines sériques du zébu arabe du Tchad, Rev. Elev. Méd. vét. Pays trop., 1959, 12 (3): 293-96.

7. ROUMEGOUX (J.), DOUTRE (M.P.), Etude immunoélectrophorétique du sérum de zébu Gobra au Sénégal. Possibilité de variations saisonnières qualitatives, Rev. Elev. Méd. vét. Pays trop., 1971, 24 (2) : 203-13. 